\title{
Longitudinal expression profiling of CD4+ and CD8+ cells in patients with active to quiescent giant cell arteritis
}

Elisabeth De Smit ${ }^{1 *+}$ (D) Samuel W. Lukowski ${ }^{2+}$, Lisa Anderson ${ }^{3}$, Anne Senabouth $^{2}$, Kaisar Dauyey ${ }^{2}$, Sharon Song ${ }^{3}$, Bruce Wyse ${ }^{3}$, Lawrie Wheeler ${ }^{3}$, Christine Y. Chen ${ }^{4}$, Khoa Cao ${ }^{4}$, Amy Wong Ten Yuen ${ }^{1}$, Neil Shuey ${ }^{5}$, Linda Clarke ${ }^{1}$, Isabel Lopez Sanchez ${ }^{1}$, Sandy S. C. Hung ${ }^{1}$, Alice Pébay' ${ }^{1}$ David A. Mackey ${ }^{6}$, Matthew A. Brown ${ }^{3}$,

Alex W. Hewitt ${ }^{1,7+}$ and Joseph E. Powell ${ }^{2+}$

\begin{abstract}
Background: Giant cell arteritis (GCA) is the most common form of vasculitis affecting elderly people. It is one of the few true ophthalmic emergencies but symptoms and signs are variable thereby making it a challenging disease to diagnose. A temporal artery biopsy is the gold standard to confirm GCA, but there are currently no specific biochemical markers to aid diagnosis. We aimed to identify a less invasive method to confirm the diagnosis of GCA, as well as to ascertain clinically relevant predictive biomarkers by studying the transcriptome of purified peripheral CD4+ and CD8+ T lymphocytes in patients with GCA.

Methods: We recruited 16 patients with histological evidence of GCA at the Royal Victorian Eye and Ear Hospital, Melbourne, Australia, and aimed to collect blood samples at six time points: acute phase, 2-3 weeks, 6-8 weeks, 3 months, 6 months and 12 months after clinical diagnosis. CD4+ and CD8+ T-cells were positively selected at each time point through magnetic-assisted cell sorting. RNA was extracted from all 195 collected samples for subsequent RNA sequencing. The expression profiles of patients were compared to those of 16 age-matched controls.

Results: Over the 12-month study period, polynomial modelling analyses identified 179 and 4 statistically significant transcripts with altered expression profiles (FDR $<0.05)$ between cases and controls in CD4+ and CD8+ populations, respectively. In CD8+ cells, two transcripts remained differentially expressed after 12 months; SGTB, associated with neuronal apoptosis, and FCGR3A, associatied with Takayasu arteritis. We detected genes that correlate with both symptoms and biochemical markers used for predicting long-term prognosis. 15 genes were shared across 3 phenotypes in CD4 and 16 across CD8 cells. In CD8, IL32 was common to 5 phenotypes including Polymyalgia Rheumatica, bilateral blindness and death within 12 months.

Conclusions: This is the first longitudinal gene expression study undertaken to identify robust transcriptomic biomarkers of GCA. Our results show cell type-specific transcript expression profiles, novel gene-phenotype associations, and uncover important biological pathways for this disease. In the acute phase, the gene-phenotype relationships we have identified could provide insight to potential disease severity and as such guide in initiating appropriate patient management.
\end{abstract}

Keywords: Giant cell arteritis, Disease biomarkers, RNA sequencing, Expression profiling, Transcriptome, CD4 \& CD8 T lymphocytes, Magnetic-assisted cell sorting

\footnotetext{
* Correspondence: elisabethdesmit@gmail.com

${ }^{\dagger}$ Elisabeth De Smit, Samuel W. Lukowski, Alex W. Hewitt and Joseph E. Powell contributed equally to this work.

${ }^{1}$ Centre for Eye Research Australia, The University of Melbourne, Royal

Victorian Eye \& Ear Hospital, 32 Gisborne Street, East Melbourne 3002,

Australia

Full list of author information is available at the end of the article
}

(c) The Author(s). 2018 Open Access This article is distributed under the terms of the Creative Commons Attribution 4.0 International License (http://creativecommons.org/licenses/by/4.0/), which permits unrestricted use, distribution, and reproduction in any medium, provided you give appropriate credit to the original author(s) and the source, provide a link to the Creative Commons license, and indicate if changes were made. The Creative Commons Public Domain Dedication waiver (http://creativecommons.org/publicdomain/zero/1.0/) applies to the data made available in this article, unless otherwise stated. 


\section{Background}

Giant Cell Arteritis (GCA) is the most common form of vasculitis in people over 50 years of age, and has a predilection for medium- and large-sized vessels of the head and neck. GCA represents one of the few true ophthalmic emergencies, and given the severe sequelae of untreated disease, a timely diagnosis is crucial [1]. GCA is a devastating disease associated with significant morbidity and mortality. If untreated, GCA can cause catastrophic complications including blindness and stroke, as well as aortic dissection and rupture.

The patho-aetiology of GCA is poorly understood. It is likely that both a genetic predisposition and possible environmental factors, the latter unconfirmed, contribute to the onset of disease [2]. GCA is a heterogenous disease and a definitive diagnosis can be difficult to establish in the acute setting. The current gold standard for diagnosis is a temporal artery biopsy, which is an invasive surgical procedure $[3,4]$. There are currently no specific biomarkers to diagnose GCA, or stratify patient management.

In the acute setting, treatment with high-dose corticosteroids should be started empirically when a patient's symptoms and/or inflammatory markers suggest a diagnosis of GCA is likely [1]. Treatment should not be delayed whilst waiting for biopsy results to become available. Once diagnosed, clinicians monitor disease activity based on patients' symptoms and inflammatory markers, primarily the erythrocyte sedimentation rate (ESR) and C-reactive protein (CRP). However, these biochemical markers are nonspecific and may be elevated in other inflammatory or infective diagnoses. There is a pressing need for more sensitive and specific biomarkers. This would aid in making a diagnosis, as well managing this condition more appropriately and mitigate the need for an invasive surgical procedure. Motivated by this need, we aimed to discover a biomarker so that when patients present to the emergency department with features of GCA, a blood test could be performed, allowing prompt diagnosis and initiation of appropriate treatment.

GCA is presumed to be an autoimmune disease with a highly complex immunopathogenesis. It has a strong association with HLA class II suggesting an adaptive immune response with antigen presentation to CD4+ T cells [5]. CD8+ T cells have also been described in GCA both at tissue level and peripherally [6, 7]. Transcriptional profiling in blood consists of measuring RNA abundance in circulating nucleated cells. Changes in transcript abundance can result from exposure to host- or pathogen-derived immunogenic factors. Given that $\mathrm{T}$ Lymphocytes are key mediators of the adaptive cellular immune response and in GCA [8], we studied the transcriptome of peripheral CD4+ and CD8+ T cells of patients with GCA. We monitored patients' expression profiling along the course of their disease to detect changes in transcripts as disease state altered and became quiescent.

\section{Methods}

\section{Patient recruitment}

Between July 2014 and June 2016, 16 patients presenting to the emergency department (ED) at the Royal Victorian Eye \& Ear Hospital (RVEEH) in Melbourne (Australia), with symptoms and signs consistent with the diagnosis of GCA were enrolled in our study (Fig. 1). Ethics was approved for this study through the RVEEH (Ethics $11 / 998 \mathrm{H}$ ), and all patients provided informed written consent to participate in serial sample collections, and for publication of results. We acquired blood samples from patients in the acute phase of their disease T1 (Day 0-7) but ideally prior to steroid initiation. Analysis took into account those patients who were steroid-naive at T1 and those who had already started steroid treatment, albeit in some cases less than $24 \mathrm{~h}$ earlier. In addition to $\mathrm{T} 1$, we aimed to acquire five subsequent serial samples from each patient - T2 (2-3 weeks), T3 (68 weeks), T4 ( 3 months), T5 ( $\sim 6$ months $)$ and T6 ( 12 months) after presentation - to detect changes in their transcripts as the disease state altered and became quiescent (Additional file 1: Table S1). For each patient with GCA, we recruited an age- and gender-matched healthy control from whom two serial blood samples were collected 2-3 weeks apart. Our study design is outlined in Fig. 1.

\section{T-cell isolation}

At each visit, $36 \mathrm{ml}$ of peripheral blood were collected in $4 \times 9 \mathrm{ml}$ ethylenediaminetetraacetic acid (EDTA) tubes, $18 \mathrm{ml}$ of which were used to isolate each of the two T-cell populations. Once blood was collected from a patient, it was processed within $30 \mathrm{~min}$. Rapid processing was conducted to avoid changes in cellular expression profiles [9]. First, the peripheral blood mononuclear cells (PBMCs) were isolated using Ficoll-Paque density centrifugation. This was followed by positive selection with magnetic antibody-coupled microbeads (MACS) (CD4 Human Microbeads (130-045-101) and CD8 Human Microbeads (130-045-201) from Miltenyi Biotec), to isolate the CD4+ and CD8+ T-cell populations from PBMCs. CD4+ cells were labelled with fluorescein isothiocyanate (CD4-Viobright FITC (130-104-515) Miltenyi Biotec) and CD8+ with allophycocyanin (CD8-APC (130-091-076) Miltenyi Biotec) antibody for purity analysis. The CD4+ and CD8+ positive fractions were eluted from the magnetically charged MS column in 1000ul of MACS BSA Stock Solution 1:20 with autoMACS Rinsing Solution (Miltenyi Biotec). A $20 \mu \mathrm{l}$ aliquot of both CD4+ and CD8+ final cell populations was fixed in $2 \%$ paraformaldehyde (PFA) and used for analysis of the population purity on a CyAn ADP fluorescence-activated cell sorting (FACS) analyzer (Additional file 2: Fig. S1). The remainder of the positive fractions was stored at $-80{ }^{\circ} \mathrm{C}$ in lysis RLT buffer (Qiagen) to which beta-mercaptoethanol had been added as per manufacturer's guidelines for between 1 and 23 months. 


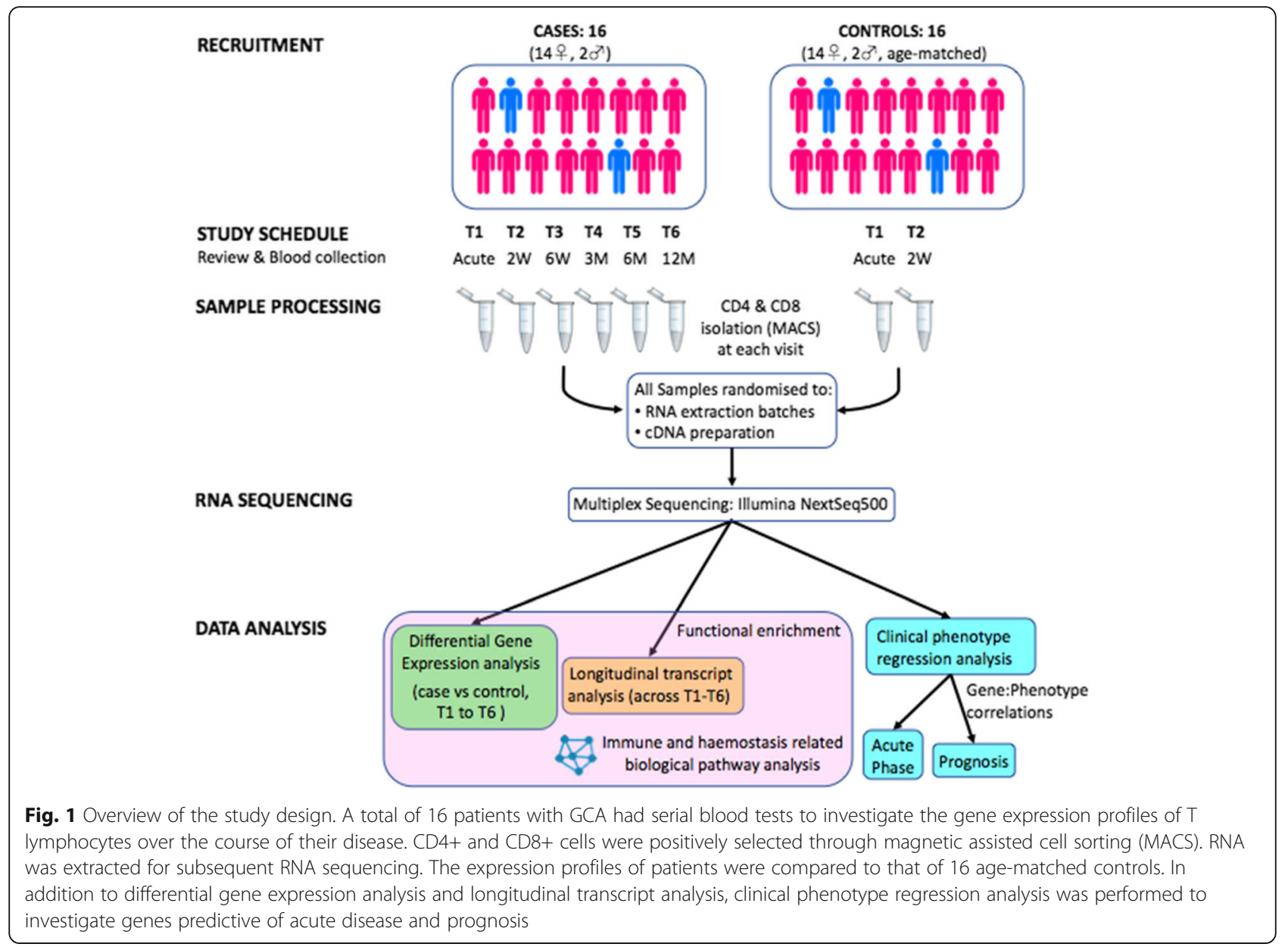

RNA extraction, cDNA processing and RNA sequencing

T cell samples underwent RNA extraction as per manufacturer's protocol (Qiagen RNeasy kit) at the Centre for Eye Research Australia (CERA) located in the Royal Victorian Eye and Ear Hospital. All T-cell lysate samples, 135 GCA patient samples and 60 control samples, were randomised to RNA extraction batches of between 20 and 24 samples to avoid batch effects. RNA samples were eluted $30 \mu \mathrm{l}$ in RNAse free water and stored at $-80{ }^{\circ} \mathrm{C}$ until all extractions were complete. Samples were tested on the NanoDrop ND-1000 spectrophotometer to check RNA quantity and quality (A260/A230 and A260/A280 between 1.8 and 2.1). Once all batches were extracted, samples were dispatched on dry ice to the Australian Translational Genomics Centre (ATGC) at Queensland University of Technology (QUT) for cDNA processing and RNA sequencing. At ATGC, RNA integrity (RIN) and quantity was confirmed with a Bioanalyzer 2100 (Agilent) before undergoing library preparation.

To avoid sequencing batch effects, all 195 samples (GCA $n=135$, and Control $n=60$ ) were re-randomised to be processed in one of three different cDNA library preparation batches (Illumina TruSeq Stranded mRNA
Sample Preparation Kits). This kit purifies the polyadenylated mRNA molecules. The Illumina Truseq protocol is optimized for $0.1-4 \mu \mathrm{g}$ of total RNA and a RIN value $\geq 8$ is recommended. The average total RNA yield varied between samples. The average RNA concentration was $137.9 \mathrm{ng} / \mu \mathrm{l}$ (range 12.1 to $1130.0 \mathrm{ng} / \mu \mathrm{l}$ ). Total RNA yield per sample averaged to $2757.7 \mathrm{ng}$ (range 242.0 to $22,600.0 \mathrm{ng}$ ) and average RIN was 8.9 (range 7.2 to 10.0 ). 600 ng total RNA was used to generate cDNA libraries (30 $\mu \mathrm{l}$ ) for all samples with $\geq 600 \mathrm{ng}$ total RNA available. Samples with less than $600 \mathrm{ng}$ total RNA available were used entirely. Samples were barcoded to allow large throughput at sequencing. The number of PCR cycles for cDNA amplification was adjusted as required to equalise the cDNA yield as per the protocol. Quality control of library concentrations was assessed through LabChip GX High Sensitivity DNA assay.

RNA-Seq libraries were multiplexed and sequenced (75 bp PE) in batches on an Illumina NextSeq500 high-throughput instrument. Each batch of cDNA libraries was pooled in equimolar volumes, and sequenced over three flow cells, with nine flow cells used in total. To achieve uniform sequencing across a large number of 
samples, the data were reviewed following each run by determining the number of mapped reads per sample. The read count per sample volume pooled was used as a metric to re-pool the cDNA libraries for additional sequencing. As such the pool of cDNA libraries for each batch was adjusted so that all samples would reach 16 million raw reads. This strategy also minimised between sample sequence run batch effects. cDNA libraries were sequenced and we obtained a median 11,017,433 mapped reads per sample and the read counts were aggregated into a single gene expression matrix. 40,744 transcripts had counts-per-million $(\mathrm{cpm})>1$ in $50 \%$ of samples and underwent further analysis.

\section{Computational analysis}

Quality control of the sequencing data was performed on the FASTQ files. High quality reads were retained and Trimmomatic v0.36 was used to remove adapters and low quality bases. Reads were mapped to the GRCh38 human reference transcriptome using Kallisto v0.42.4 [10]. Only those with cpm $>1$ in $50 \%$ of the samples were retained for further analysis. Transcript expression between libraries was normalised using the trimmed mean of $\mathrm{M}$ method (TMM) and corrected for batch effects using the removeBatchEffect function implemented in edgeR (Flowcell ID, Gender and Ethnicity) [11]. Hierarchical clustering and principal component analysis (PCA) confirmed the absence of batch effects and outlier samples (Additional file 3: Figure S2).

\section{Differential gene expression analysis}

A total of 135 GCA samples ( $n=16$ patients) spanning six timepoints and 60 control samples ( $n=16$ patients) spanning two timepoints were grouped for analysis based on their $\mathrm{CD} 4(\mathrm{GCA}=68$, control $=30)$ or $\mathrm{CD} 8$ MACS $(G C A=67$, control $=30)$ separation. This grouping strategy formed the basis of the differential expression design matrix, allowing pairwise comparisons between individual timepoints on a case/control or CD4/CD8 basis. Differentially expressed transcripts were considered statistically significant if their false discovery rate (FDR) was less than 0.05. Differential expression (DGE) analysis between case and control subjects was performed comparing the initial $\mathrm{T} 1$ case specimens versus both the T1 and T2 of control specimens. Transcripts below FDR $<0.05$ and a two-fold change between cases and controls were considered significant.

\section{Polynomial modelling of transcript expression}

The longitudinal expression profile of retained transcripts across six time points was tested for significant changes using polynomial regression. Polynomial regression modelling was performed with the patient weight-normalised steroid dosage fitted as a fixed effect. Steroid dose was normalised by dividing the Daily Steroid Dose by the Patient Weight. The global model $p$-value was corrected for multiple testing using the Benjamini-Hochberg method (FDR) and transcripts with an adjusted $p$-value below the FDR threshold $(<0.05)$ were considered statistically significant.

\section{Functional enrichment and pathway analysis}

Functional enrichment analysis was performed using the Reactome biological pathway database via the ReactomePA software package (version 1.18) and the CPdB web server (http://cpdb.molgen.mpg.de/) [12]. Pathway analysis results with adjusted $p$-values below the FDR threshold $(<0.1)$ were considered significant.

\section{Clinical phenotype regression analysis}

Models were constructed to regress clinically relevant traits that were measured at the time of disease onset, or sample collection, against normalised gene expression levels. For quantitative clinical variables we used a linear model, and for categorical variables we used a logistic regression model. Clinical phenotypes were fitted against the expression of each of transcripts in GCA-only samples separated into $\mathrm{CD} 4+$ and $\mathrm{CD} 8+$ populations and weight-normalised daily steroid dose was included as a fixed effect. For each transcript, the adjusted $p$-value was calculated using the Benjamini-Hochberg method (FDR) method [13]. Transcripts with adjusted $p$-values below the FDR threshold $(<0.01)$ were retained for further analysis. The complete summary tables of tested phenotypes are available in Tables 2, 3 and 4.

\section{Results}

Patient recruitment and MACS events

Sixteen incident patients with active GCA and 16 age-matched controls were recruited. The mean age was 78.2 years in the GCA cohort and 76.6 years in the control group. Both groups had the same 14:2 female to male ratio. Table 2 provides the number of patients presenting with the common symptoms and signs associated with GCA. Additional file 4: Table S2 and Additional file 5: Table S3 describe the specific ophthalmic manifestations and long-term prognoses observed in our patient cohort. Not all patients were able to complete 12 months of participation; therefore, not all patients had six samples collected (Additional file 1: Table S1). 6 patients were steroid-naive at $\mathrm{T} 1$; these patients had their first sample collected in the ED prior to commencing steroid treatment. Of the other $10 \mathrm{pa}$ tients, 3 patients had been on steroids less than $24 \mathrm{~h}$, and the other 7 patients had been on steroids for between three to seven days at the time of $\mathrm{T} 1$.

In total, 195 MACS events, comprising 135 GCA (67 CD4 and 66 CD8 samples) and 60 control events, were performed (Additional file 1: Table S1). One patient's CD8 sample had insufficient material after isolating the PBMC 
layer and was therefore excluded from further analysis (Additional file 1: Table S1). Two controls were only able to provide the first time point samples. Each MACS procedure isolated between 2 and 10 million CD4+ and CD8+ cells per patient per time event. CD4+ MACS isolation resulted in greater cell counts than CD8+. The analysis on the CyAn ADP analyser revealed good population purity after MACS-positive cell selection: an average of $97 \%$ for CD4+ cells and > 94\% for CD8+ cells (Additional file 2: Figure S1).

\section{Differential expression analysis:}

To determine which transcripts showed the most variation in expression over the 12-month collection period, and to identify cell type specific signatures, we analysed the expression levels of samples from GCA patients $(n=135)$ (Additional file 6: Figure S3). Figure 2 represents the expression levels of the top 40 most variable transcripts in CD4+ and CD8+ samples in GCA patients. The expression levels of control genes such as $C D 4$ and $C D 8 A / B$ confirms the partitioning of CD4+ and CD8+ cells.
We investigated changes in gene expression in both CD4 + and CD8+ between cases and controls at T1. At a significance threshold of $\mathrm{FDR}<0.05$, we identified 67 down-regulated (DR) and 129 up-regulated (UR) transcripts in CD4+ samples, and 93 DR and 188 UR transcripts in CD8+ samples (Table 1). The numbers of significantly differentially expressed transcripts increased dramatically at $\mathrm{T} 3$ in cases compared to the controls at T1 for CD8+ samples, and resolving to a near-control profile at T6. At T3 (6-8 weeks), we detected 1927 DR and 1783 UR transcripts in CD8+ cells. Interestingly, DE transcripts in CD4+ cells reached a plateau from T2 to T4 (T2: 254 DR/228 UR; T3: 196 DR/190 UR; T4: 179 DR/200 UR).

We hypothesised that gene expression in GCA patients would return to baseline levels at approximately 12 months, corresponding to T6, marking disease quiescence. Transcripts remaining DE at T6 may be of clinical interest or mark evidence of previous disease despite current inactivity. In CD8+ cells, we identified two significant DE transcripts at T6 versus controls, SGTB (Small glutamine-rich tetratricopeptide repeat (TPR)-containing beta) and FCGR3A (Fc Fragment Of IgG Receptor IIIa),

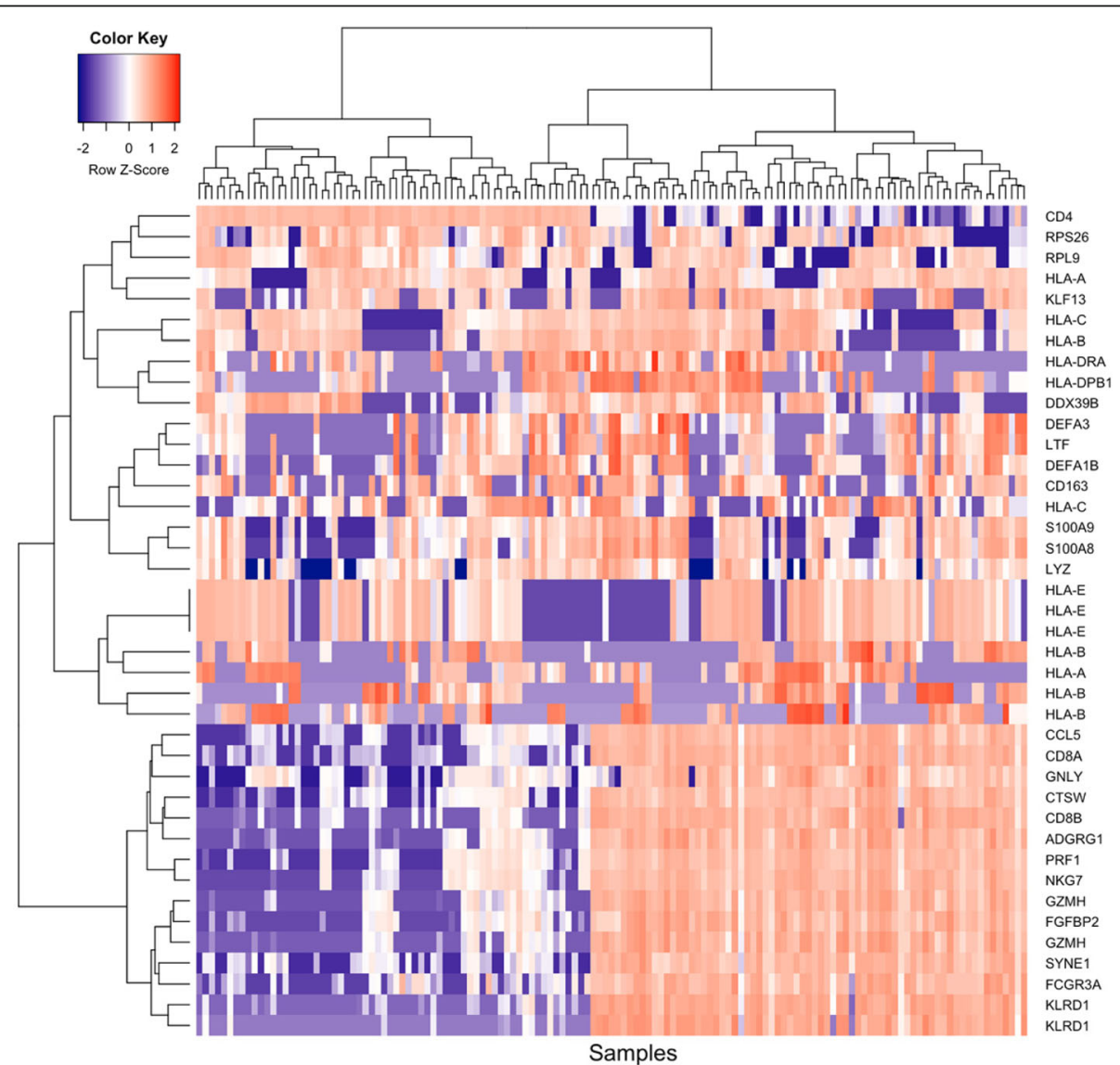

Fig. 2 Expression levels of the top 40 genes with highest expression variation in CD4 and CD8 samples for all GCA patients. The color scale indicates normalised, $\log _{2}$-transformed gene expression (cpm), from low (blue) to high (red). Multiple gene IDs represent alternative transcript isoforms 
Table 1 Number of DE genes in each comparison

\begin{tabular}{llllll}
\hline & CD4 & & & CD8 \\
\cline { 2 - 3 } \cline { 5 - 6 } Contrast & DR & UR & & DR & UR \\
\hline Control 2 vs Control 1 & 0 & 0 & 0 & 0 \\
GCA T2 vs T1 & 0 & 0 & 0 & 0 \\
GCA T3 vs T1 & 1 & 8 & 35 & 80 \\
GCA T4 vs T1 & 2 & 7 & 1 & 3 \\
GCA T5 vs T1 & 0 & 0 & 0 & 0 \\
GCA T6 vs T1 & 0 & 0 & 2 & 0 \\
GCA T6 vs T3 & 0 & 0 & 45 & 10 \\
GCA T1 vs Control 1 & 67 & 129 & 93 & 188 \\
GCA T2 vs Control 1 & 254 & 228 & 325 & 453 \\
GCA T3 vs Control 1 & 196 & 190 & 1927 & 1783 \\
GCA T4 vs Control 1 & 179 & 200 & 576 & 827 \\
GCA T5 vs Control 1 & 1 & 1 & 101 & 296 \\
GCA T6 vs Control 1 & 0 & 0 & 1 & 1 \\
GCA T1 vs Control 2 & 22 & 58 & 58 & 156 \\
GCA T2 vs Control 2 & 276 & 233 & 187 & 335 \\
GCA T3 vs Control 2 & 194 & 171 & 1066 & 1227 \\
GCA T4 vs Control 2 & 197 & 179 & 351 & 615 \\
GCA T5 vs Control 2 & 2 & 0 & 55 & 222 \\
GCA T6 vs Control 2 & 0 & 0 & 0 & 0 \\
\hline
\end{tabular}

which showed $\log _{2}$ fold changes in expression of -0.54 $\left(p=4.83 \times 10^{-7}\right)$ and $1.99\left(p=1.75 \times 10^{-6}\right)$, respectively. There were no significant DE transcripts in the CD4+ cells between GCA T6 and the controls.

Differentially expressed genes between $\mathrm{T} 1$ and $\mathrm{T} 6$ in GCA patients could represent a biomarker of disease activity, marking either gene UR or DR during the acute phase of disease and then normalising as disease quiesces. From the CD8+ cell analysis, we detected two differentially expressed isoforms of CD163 with significantly reduced expression levels. At T6 compared to T1, CD163 isoform 1 (ENST00000359156) expression showed a $\log _{2}$ fold change (FC) of $-6.01\left(p=1.07 \times 10^{-}\right.$ $\left.{ }^{6}\right)$, whereas the $\log _{2}$ FC of CD163 isoform 2 (ENST00000432237) was $-9.69\left(p=5.84 \times 10^{-8}\right)$. Notably, CD163 expression is suppressed in response to pro-inflammatory stimuli in monocytes [14], and is inversely correlated with $C D 16$ expression $[14,15]$, which is consistent with the increased $C D 16$ expression we observed in cases compared to controls at T6 (12 months). However, CD16 was not consistently differentially expressed across all time points in CD8+ cells. There were no significant $\mathrm{DE}$ transcripts in the CD4+ cells between GCA T1 and T6. Reassuringly, no significant transcripts were observed in either CD4+ or CD8+ cells in the controls between T1 \& T2. Tables of significant differentially expressed transcripts are presented in Additional file 7: Table S4 (CD4) and Additional file 8: Table S5 (CD8).

\section{Polynomial modelling of longitudinal transcript expression:}

To identify important transcripts whose expression levels vary across a 12-month period of the study, we used polynomial regression to model changes in the expression levels of 40,744 transcripts separately in CD4+ and CD8+ cells across the six time points. Using this approach, we detected 179 and 4 statistically significant expression profiles (FDR $<0.05$ ) in $\mathrm{CD} 4+$ and $\mathrm{CD} 8+$ populations, respectively. Tables of significant transcript expression models are available in Additional file 9: Table S6.

The top $12 \mathrm{CD} 4+$ profiles and all 4 significant $\mathrm{CD} 8+$ profiles are shown in Fig. 3. In CD4+, the majority of genes demonstrated a pattern of decreased expression over the study course. Only two genes demonstrated a positive fold change and increase in expression levels over the 12 months, namely FOXO1 involved in blood vessel development and TRBC2 involved in complement cascade activation and phagocytosis. The four identified genes in CD8+ were CCLN2, FANCA, PTCD2 and THRAP3. The first three genes demonstrate a negative $\log _{2}$ fold change, whilst THRAP3 demonstrates an increased expression trend.

No substantial contribution of steroid dose to the model was observed across the 12-month time course (CD4: median beta $=-0.001$, median $p=0.439$; CD8: median beta $=-0.002$, median $p=0.463)$. However, expression levels of certain genes at $\mathrm{T} 1$ may have been affected depending on whether patients were steroid-naive or had already been started on treatment at time of their first blood sample collection. Figures $3 \mathrm{a}$ and $\mathrm{b}$ highlight those patients who were steroid-naive in red and those who had already been started on steroid treatment in black. Expression of certain genes, for example TIMD4, VIPR1, and FOXO1, show obvious clustering depending on a patient's treatment status and appear to be affected by corticosteroid initiation. Steroid treatment, even though only initiated in some instances less than $24 \mathrm{~h}$ prior to blood collection at $\mathrm{T} 1$, has a clear effect on the expression of certain genes.

In CD4+, three genes, LMBR1L, UAP1L1 and $K C N M B 4$, showed least clustering at $\mathrm{T} 1$ and appeared least affected by steroid treatment, albeit having been through oral dose or intravenously administered prior to T1 collection. In CD8+ cells, PTCD2 and THRAP3 appear little affected by steroids at T1. PTCD2 is highly expressed in both steroid-naive and patients on steroids at T1 and less so at T6, suggesting no major influence of steroids at T1. THRAP3 shows increased expression over time suggesting that in the acute phase THRAP3 expression might be suppressed. 


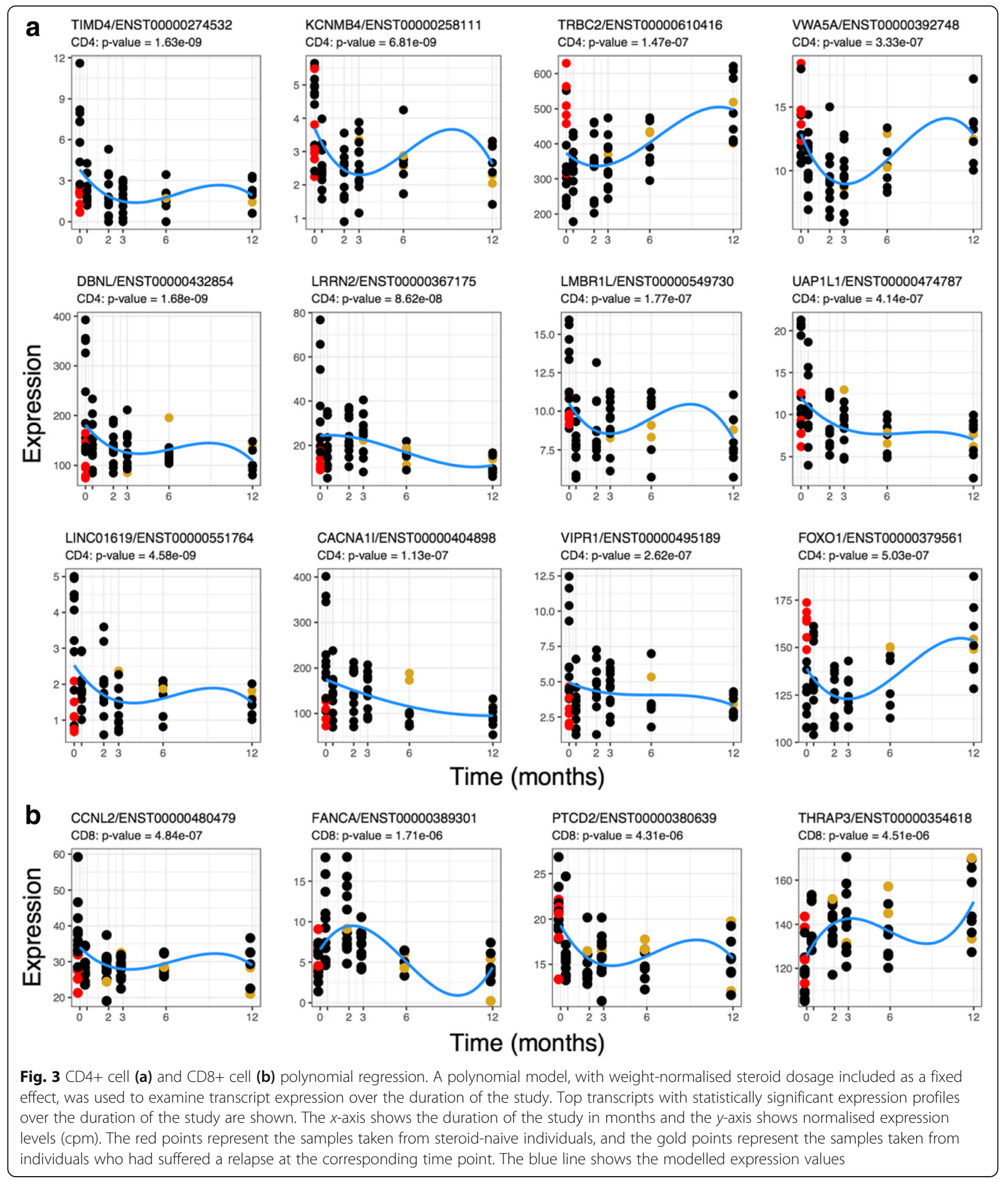

From our DGE analysis, we observed significant reduction in CD163 transcript expression between T1 and T6 in the CD8 cell population analysis. Our results for the polynomial expression modelling also reflected that CD163 was significantly reduced at T6. However, model profiles of this transcript showed that the trend over the 12 -month time course was not statistically significant (FDR > 0.05). Interestingly, we noted that several CD163 isoforms in the analyses of both CD4+ and CD8+ cell populations had compelling model profiles. For all but 


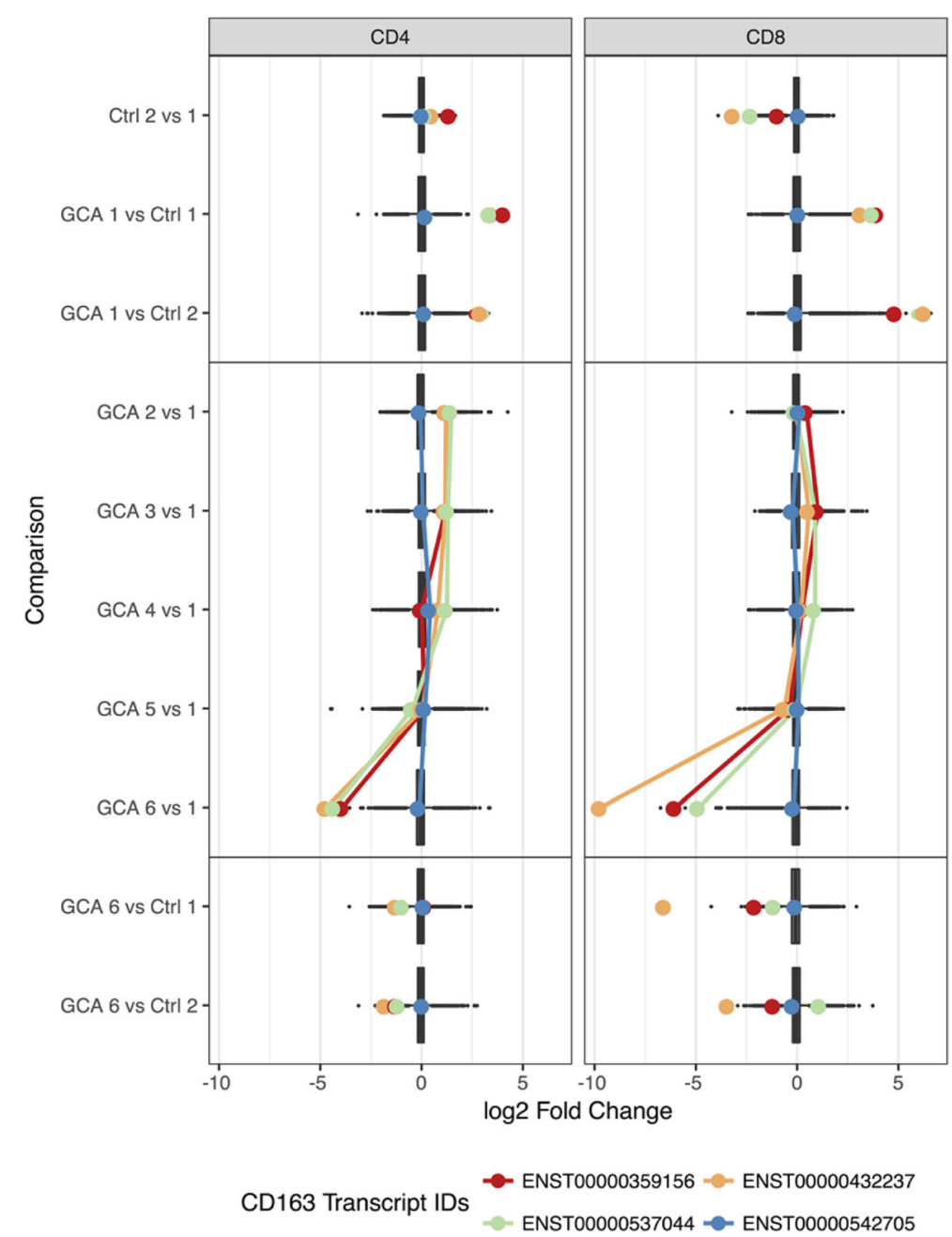

Fig. 4 Fold-change distribution of differentially expressed transcripts in CD4 and CD8 samples for each differential expression comparison. Coloured points indicate the $\log _{2}$ foldchange of CD163 expression and shown for each transcript in CD4 and CD8 samples. Lines connect the foldchange values ( $\log _{2}$-transformed) of differential expression comparisons along the time course only

one CD163 isoform, expression levels returned to zero for all individuals at 12 months; however, these were not FDR-significant. The $\log _{2}$ foldchange in the expression of these transcripts over 12 months is shown in Fig. 4.

\section{Functional enrichment and pathway analysis:}

For individuals with GCA, we would expect an enrichment of immune and inflammation related pathways compared to healthy individuals. Biological pathway analysis of differentially expressed transcripts and statistically significant transcripts identified in the polynomial expression modelling analysis was performed using the curated Reactome database.

Significant DE transcripts in CD4+ samples comparing GCA to controls in the early time points showed a significant enrichment of $\mathrm{T}$-cell receptor signaling (adj. $p$-value $=4.25 \times 10^{-3} ; 11$ genes). In CD8+ samples, we observed an enrichment of genes in pathways related to platelet degranulation (adj. $p$-value $=0.0124 ; 12$ genes) and activation (adj. $p$-value $=0.0156 ; 20$ genes), as well as Fc-gamma receptor (FCGR) dependent phagocytosis (adj. $p$-value $=0.0156 ; 13$ genes). Furthermore, CD8+ samples from first two collected samples of GCA cases showed significant enrichment of pathways related to haemostasis (adj. $p$-value $=2.63 \times 10^{-6} ; 118$ genes), innate immune system (adj. $p$-value $=5.51 \times 10^{-6} ; 169$ genes) and the adaptive immune system (adj. $p$-value $=$ $3.24 \times 10^{-4} ; 129$ genes).

Transcripts with a significant association across the 12-month collection time were interrogated for enrichment of specific biological pathways. We tested all 179 CD4 and 4 CD8 significant transcripts. In the CD4 transcripts, we observed an over-representation of transcripts in the integrin cell surface interactions (adj. $p$-value $=0.015)$ and Caspase-mediated cleavage of cytoskeletal proteins (adj. $p$-value $=0.0325$ ) as well as 
cytokine signaling (adj. $p$-value $=0.08)$ and negative regulators of RIG-I/MDA5 signaling (adj. $p$-value $=0.08$ ). In the CD8 results, there were insufficient significant transcripts to perform enrichment analyses. However, a literature search revealed THRAP3 is involved in intracellular steroid hormone receptor signaling pathways, and FANCA in inflammatory responses and T-cell differentiation pathways.

\section{Clinical phenotype regression analysis:}

Linear and logistic regression models were used to estimate the effect of specific clinically important phenotypes on expressed transcripts. The analyses were three-fold. The first was to determine whether there were any genes that correlated with symptoms and signs used in the acute setting (T1) (Table 2). Second, we determined whether any genes directly correlated with the biochemical markers currently used in the acute phase (T1) (Table 3). Genes resulting from these first two analyses are potential biomarkers for disease activity in the acute setting and predict relapses. Thirdly, we determined gene correlations with markers of disease severity or prognosis (Table 4). These were categorised in terms of visual outcome: whether blinded in one eye, "monocular", or both eyes, "bilateral"; relapse events; and whether the patient died during the study period. This enables us to identify genes that could provide prognostic information, ideally at the time of diagnosis (T1) but also during the course of disease (T1-6).

\section{Correlation with clinical features in the acute setting}

At the time of admission (T1), we would expect to observe some changes in gene expression to be strongly associated with clinical phenotypes related to the acute onset of disease. To identify a transcriptional signature that may be specific to active GCA, we examined the effect of clinically relevant phenotypes on gene expression
Table 3 "Acute phase" biochemical markers

\begin{tabular}{llll}
\hline & Phenotype & CD4 & CD8 \\
\hline 1 & ESR & 23 & 15 \\
2 & CRP & 12 & 15 \\
3 & Platelets & 41 & 7 \\
4 & WCC & 75 & 38 \\
5 & Lymphocytes & 23 & 63 \\
6 & Neutrophils & 22 & 133 \\
\hline
\end{tabular}

Number of genes significantly affected (FDR $<0.01)$ by biochemical markers in regression models at $\mathrm{T} 1$

in CD4 and CD8 samples taken at T1. Table 2 lists the eleven phenotypes and the number of statistically significant transcripts $(\mathrm{FDR}<0.01)$ observed for each in CD4 or CD8 samples at T1. Genes or transcripts that are common to multiple symptoms/signs are likely to be clinically relevant, particularly at the acute onset of disease. In CD4 and CD8 samples, we identified 17 (CD4) and 27 (CD8) transcripts that were significantly associated with two or more clinical phenotypes.

In CD4 cells, LAMTOR4 is a gene shared between jaw claudication and temporal headache, two important clinical features in acute GCA. Another gene associated with jaw claudication is $G Z M B$, which is also associated with visual disturbance. PPP1CB and EIF4A3 were shared by both jaw claudication and a background history of Polymyalgia Rheumatica (PMR). EXTL3, was expressed in both patients with jaw claudication and fatigue. We identified numerous genes associated with headache, both temporal and other types: POFUT2 in CD4 cells, and SLC35F6, HTD2, ZNF708, KLRC4-KLRK1 and JMJD7 in CD8 cells. EIF5A in CD8 cells was common to both malaise and temporal headache. SLA and ETS1 are genes shared by patients with a history of PMR diagnosis and those experiencing visual disturbances at $\mathrm{T} 1$.

Table 2 "Acute phase" symptoms, signs and relevant past medical history

\begin{tabular}{lllll}
\hline & Phenotype & Number of patients with & \multicolumn{2}{l}{ Number of transcripts per cell type correlating to each phenotype } \\
\cline { 4 - 5 } & & each feature at time of presentation & CD4 & 247 \\
\hline 1 & Visual Disturbance & 14 & 23 & 34 \\
3 & Temporal Headache & 14 & 67 & 76 \\
4 & Other Headache & 13 & 30 & 7 \\
5 & Scalp Tenderness & 12 & 10 & 27 \\
6 & Malaise & 12 & 8 & 10 \\
7 & Faw Claudication & 11 & 70 & 29 \\
8 & Latigue & 11 & 6 & 32 \\
9 & Weigs of Appetite & 9 & 59 & 55 \\
10 & Fever & 8 & 27 & 41 \\
11 & Polymyalgia Rheumatica & 4 & 177 & 53 \\
\hline
\end{tabular}

Number of patients (total $\mathrm{n}=16$ ) and genes significantly affected (FDR $<0.01$ ) by clinical phenotype in regression models at $\mathrm{T} 1$ 
Table 4 "Prognostic genes"

\begin{tabular}{|c|c|c|c|c|c|}
\hline & & $\mathrm{T} 1$ & & T1-T6 & \\
\hline & Phenotype & $\mathrm{CD} 4$ & CD8 & $\overline{C D 4}$ & CD8 \\
\hline 1 & Monocular Blindness & 22 & 41 & 26 & 56 \\
\hline 2 & Bilateral Blindness & 22 & 50 & 21 & 18 \\
\hline 3 & Stroke/TIA & 40 & 4 & 153 & 70 \\
\hline 4 & Relapse Events & 6 & 3 & 47 & 166 \\
\hline 5 & Deceased within 12 months & 878 & 904 & 43 & 50 \\
\hline
\end{tabular}

Number of genes significantly affected $(F D R<0.01)$ by outcome and prognostic phenotype markers in regression models both in the acute phase alone (T1) as well as across all time points (T1-T6)

Genes shared by three clinically important phenotypes at $\mathrm{T} 1$ are even more promising than those shared by two phenotypes and included 15 genes in CD4 and 16 in CD8 cells (Table 5). SRRT in CD4 was common to four phenotypes: death, fever, and both headache types. In CD8, IL32 was common to five phenotypes: visual disturbance and raised neutrophils at T1, a history of PMR, and bilateral blindness and death within 12 months (Additional file 10: Table S7 \& Additional file 11: Table S8 show shared genes per phenotype).

\section{Correlation with currently used biochemical markers}

We asked whether the results of several routine blood tests, including white cell count, platelet count, ESR and CRP correlated with changes in gene expression (Table 3). We observed significant clinical associations for each biochemical marker in both CD4 and CD8 samples.

Thrombocytosis - raised platelet count - is a good predictor of acute GCA [16]. Our analysis revealed associations of multiple genes common to both raised platelet count and fever in CD4 cells, namely $A T P 9 B, S E C 23 A, P D Z D 4$, $A B C A 2, E L K 1, C C D C 88 C$ and $D G K Z$. In addition, ESR and CRP are biomarkers commonly used to predict the likelihood of GCA, and we found that SAP18 in CD4 was associated with raised ESR and jaw claudication, whereas in CD8 cells AMPD2 was associated with raised CRP and visual disturbances.

White-blood cell count (WCC), neutrophil and lymphocyte count may also be affected in GCA, although this may be due to the corticosteroid treatment rather than the inflammatory process [17]. In the CD4 cells of our patients, we found that SPPL2B expression was common to both those with raised WCC and jaw claudication whilst MATR3 was associated with raised WCC and long-term monocular blindness. NDUFS7 expression in CD4 cells was associated with an increased lymphocyte count and temporal headache in CD4, whereas in CD8 cells AP1G2 was common to raised lymphocytes and visual disturbance. Additionally, expression of ZNF343 and INTS14 in CD4 cells were associated with both raised neutrophil and with scalp tenderness and event relapses respectively.
Correlation with prognostic outcome 12 months after diagnosis We identified genes that overlap between phenotypes marking acute disease as well as those marking prognosis. For example, temporal headache at $\mathrm{T} 1$ as well as bilateral blindness showed significant association with CD8 expression of TCF7 (TH: beta $=-0.151$, adj. $p$-value $=6.0 \times 10^{-4}$, $B B$ : beta $=-1.801$, adj. $p$-value $=2.2 \times 10^{-3}$ ) and NUCB2 (beta $=1.571$, adj. $p$-value $\left.=1.31 \times 10^{-6}\right)$. The expression of such genes could provide insight into visual prognosis in those patients presenting with headache in GCA. RPL17 in CD8 was associated between jaw claudication and relapse events, and FTSJ1 in CD4 between jaw claudication and long-term cerebrovascular events. Many genes were shared between multiple acute phase phenotypes and mortality within 12 months (Table 5). Fig. 5 shows the network analysis of clinically correlated phenotypes with shared genes, and highlights the link between phenotypes through significant shared genes.

\section{Discussion}

Through transcriptional profiling of T-lymphocytes, we identified 4031 genes in CD4+ and CD8+ cells (CD4: 884; CD8: 3147$)$ that are differentially expressed between patients with active GCA compared to age- and sex-matched controls. Longitudinal profiling of cases was undertaken with the aim of distinguishing genes that are up- or down-regulated during the acute phase of disease, which later normalise as the disease quiesces. We hypothesised that gene expression in GCA patients would return to normal at approximately 12 months. With polynomial modeling analysis of the significant differentially expressed genes, we identified 4 transcripts in CD8+ cells and 179 in CD4+ cells that show a change in expression profile over the course of twelve months (Fig. 2). As there were no statistically significant differentially expressed genes between both samples taken from controls subjects at separate times, the genes we report as differentially expressed likely represent true changes occurring in GCA disease activity.

Next, we determined whether the fold change in expression was secondary to the true effect of disease status rather than due to steroid treatment. It is important to take into consideration steroid influence on gene expression, especially early in the treatment course, as this would allow for the identification of a biomarker that could help diagnose GCA in the acute setting prior to treatment. As patients received high-dose corticosteroids between T2-T6, we compared gene expression of those patients who were steroid naive versus those who had already been initiated on treatment at their first sample collection. LMBR1L, UAP1L1 and KCNMB4 in CD4, and PTCD2 and THRAP3 in CD8, showed least clustering at the initial collection and seemed least affected by steroids at T1 (Fig. 3), suggesting that the expression profiles of these genes seen in patients, compared to 
Table 5 Genes associated with multiple phenotypes, both acute and prognostic, in CD4 and CD8 T cells

\begin{tabular}{|c|c|c|c|}
\hline Gene & Phenotype 1 & Phenotype 2 & Phenotype 3 \\
\hline \multicolumn{4}{|l|}{ CD4 } \\
\hline ATP1A1 & Temporal headache & Bilateral blindness & Death within 12 months \\
\hline LAMTOR4 & Temporal headache & Jaw claudication & Death within 12 months \\
\hline MATR3 & White cell count & Monocular blindness & Death within 12 months \\
\hline MLHI & Temporal headache & Bilateral blindness & Death within 12 months \\
\hline NDEL1 & Loss of appetite & Other headache & Death within 12 months \\
\hline NDUFS7 & Temporal headache & Elevated lymphocytes & Death within 12 months \\
\hline PDZD4 & Fever & Loss of appetite & Reduced platelets \\
\hline POFUT2 & Temporal headache & Other headache & Death within 12 months \\
\hline RRP1 & Temporal headache & Bilateral blindness & Death within 12 months \\
\hline SDCCAG3 & Bilateral blindness & Relapse events & Death within 12 months \\
\hline SEC23A & Fever & Reduced platelets & Death within 12 months \\
\hline SLCIOA3 & Fever & Reduced white cell count & Death within 12 months \\
\hline USF2 & Temporal headache & Bilateral blindness & Death within 12 months \\
\hline WDR91 & Loss of appetite & Elevated white cell count & Death within 12 months \\
\hline ZNF343 & Scalp tenderness & Reduced neutrophils & Death within 12 months \\
\hline \multicolumn{4}{|l|}{ CD8 } \\
\hline ACADVL & Elevated neutrophils & Other headache & Death within 12 months \\
\hline CD6 & Elevated neutrophils & Visual disturbance & Death within 12 months \\
\hline EIF5A & Malaise & Temporal headache & Death within 12 months \\
\hline$F D X R$ & Loss of appetite & Weight loss & Death within 12 months \\
\hline INPPLI & Malaise & Fatigue & Elevated neutrophils \\
\hline JMJD7 & Temporal headache & Other headache & Death within 12 months \\
\hline KIAA0513 & Visual disturbance & Bilateral blindness & Death within 12 months \\
\hline$K L R C 4-K L R 11$ & Temporal headache & Other headache & Death within 12 months \\
\hline MTA1 & Elevated neutrophils & Visual disturbance & Death within 12 months \\
\hline NUCB2 & Temporal headache & Bilateral blindness & Death within 12 months \\
\hline PI4KA & Elevated neutrophils & Visual disturbance & Death within 12 months \\
\hline PRAG1 & Elevated neutrophils & Bilateral blindness & Death within 12 months \\
\hline RNPS1 & Malaise & Fatigue & Death within 12 months \\
\hline SLC35F6 & Temporal headache & Other headache & Death within 12 months \\
\hline UQCRC1 & Malaise & Other headache & Death within 12 months \\
\hline ZNF708 & Temporal headache & Other headache & Death within 12 months \\
\hline
\end{tabular}

controls, is likely representative of "acute disease" at T1 rather than a steroid-induced change.

Gene expression patterns seen from our polynomial modeling analysis over the 12 months might have been influenced by systemic corticosteroid treatment (Fig. 3). In CD8+ samples, differential expression of certain genes increased dramatically at around 6-8 weeks (T3) in cases compared to the controls, and in CD4+ cells, differential expression plateaued from T2-T4. Duration of steroid treatment did not have a significant effect on expression and was removed from analysis. We also adjusted for steroid dose and patient weight in our analysis; however, the peak in expression in both cell types at these time points could be caused by a delayed or accumulation of steroid-induced effect. Nevertheless, from a diagnostic perspective, acute phase evaluation at $\mathrm{T} 1$ is most crucial for patient assessment and this potential delayed steroid-induced effect is not that problematic in our analysis. It does, however, make evaluation of expression levels in relation to relapse events between $0.5-12$ months (T2-T6) slightly challenging.

Our results show that transcripts that remain DE at 12 months (T6) could potentially be used in clinical practice to detect evidence of previous GCA disease despite 


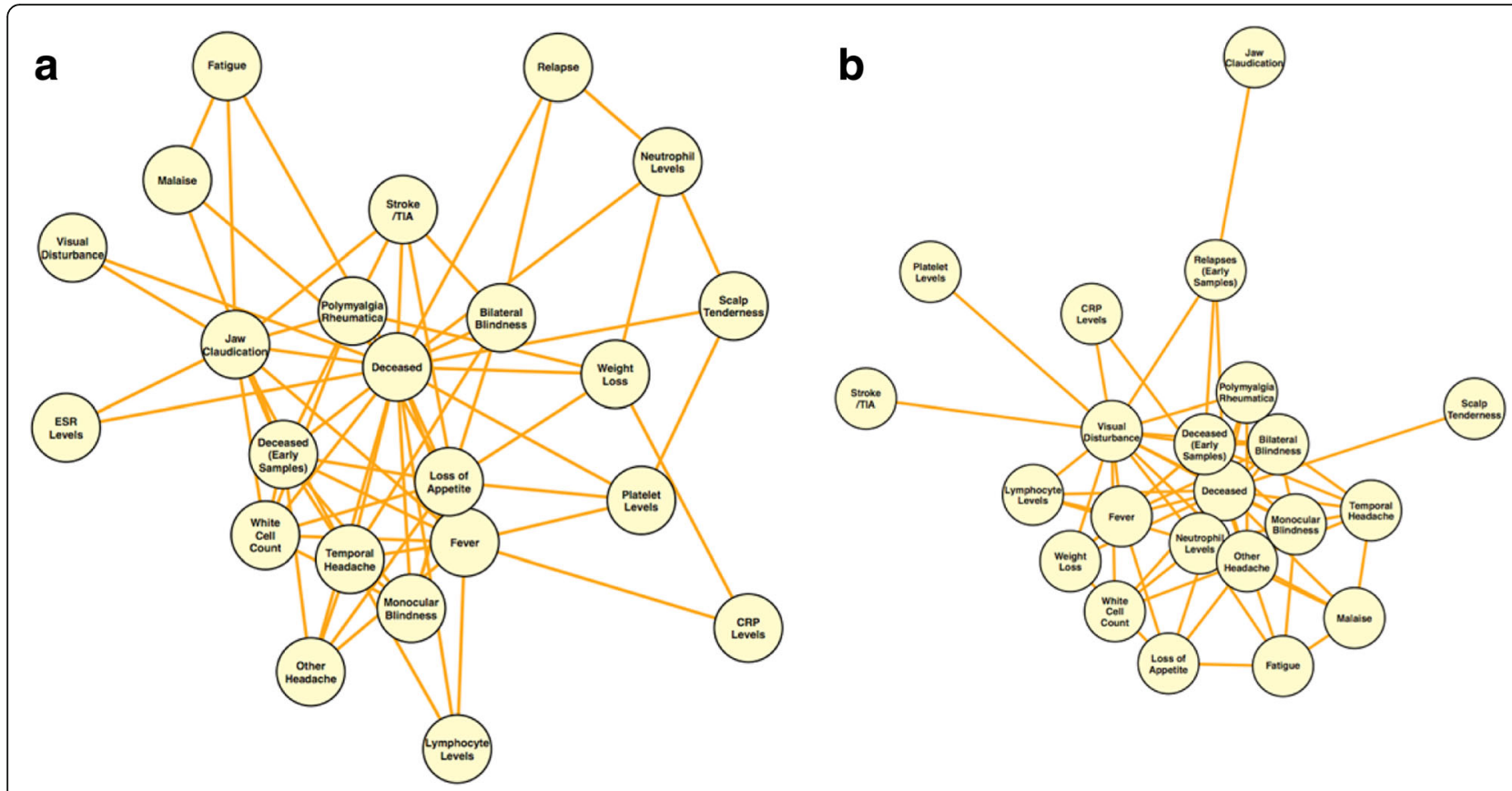

Fig. 5 Network analysis of clinically correlated phenotypes with shared genes. Network plots show the clinical phenotypes observed for GCA patients at the time of presentation with shared, statistically significant genes (FDR $<0.01)$ in (a) CD4 and (b) CD8 samples. Each network node represents a phenotype that shares significant genes with $>1$ other phenotype. Network edges represent connections (shared genes) between phenotypes

current inactivity. In CD8+ cells, we identified two significant differentially expressed transcripts at T6 versus controls, SGTB and FCGR3A. Little is known about SGTB but it has been associated with neuronal apoptosis after neuroinflammation [18]. Interestingly, FCGR3A encodes CD16a, which forms part of the Fc receptor of the immunoglobulin complex and interacts with a number of immune-related proteins including CD4 and PTPRC, a protein required for T-cell activation. Recently, Lassaunière et al. showed that Black individuals have significantly reduced proportions of FCGR3A natural killer cells $(95.2 \%$ vs. $96.9 \%)$ and CD8+ T lymphocytes (9.6\% vs. $11.7 \%$ ) compared to Caucasians [19], and this may serve as a predictive marker for a high-expressing FCGR3A phenotype in Caucasians, the population most affected by GCA. A recent genome-wide association study revealed that the FCGR2A/FCGR3A genes confer susceptibility to Takayasu arteritis, another chronic large-vessel vasculitis [20]. Furthermore, two recent studies investigating rejection in heart and kidney transplants, observed selective changes in endothelial/angiogenesis and natural killer cell transcripts, including CD16A and FCGR3A which showed increased expression with rejection phenotypes [21, 22]. Both studies illustrate the clinical potential of gene transcripts to illustrate transplant rejection diagnosis. A future study would need to be conducted to investigate the expression of FCGR3A and CD16a at the arterial level
(TAB) of GCA patients to determine whether increased expression at local level is representative to that found in peripheral T-cells. If so, FCGR3A could potentially be used as a biomarker of GCA severity in peripheral blood.

From our CD8+ cell analysis, we detected two differentially expressed isoforms of CD163 with significantly reduced expression levels at first and last collection points. $C D 163$, however, is a member of the scavenger receptor cysteine-rich (SRCR) superfamily, and is mostly expressed in monocytes and macrophages [23]. Despite an excellent T-cell population purity of $>97 \%$ isolated through MACS (Additional file 2: Figure S1), monocytes and macrophages may carry CD4+ and CD8+ cell surface markers as T lymphocytes, and may have carried over into our final positively-selected T-cell population. Irrespective of its derivative cell population, $C D 163$ expression may play a crucial role in the context of GCA and, as a result, provide crucial information. CD163 is involved in dendritic cell development, a cell crucial in the pathogenesis of GCA [24]. It has been suggested that the soluble form of $C D 163$ (sCD163) may have an anti-inflammatory role, and be a valuable diagnostic parameter for monitoring macrophage activation in inflammatory conditions where macrophage function is affected [25]. A number of clinical studies have evaluated the role SCD163 as a disease marker in inflammatory conditions including autoimmune disease, transplantation and cancer [26-28]. Expression levels of CD163 were 
reduced in our patients at T6, possibly reflecting disease quiescence. It is likely that 12 months after disease onset, the need for CD163-monocytes and macrophages to clear damaged tissue has become redundant. CD163 featured in both our differential expression and polynomial regression analyses and therefore warrants further investigation in the context of GCA, potentially through study of peripheral or tissue monocytes and macrophages.

Another strength of this study is that, through linear and logistic regression analyses, we identified associations between specific clinically important phenotypes and expressed transcripts. We detected genes which correlated with both symptoms and signs as well as biochemical markers used in the acute setting (Table 2). Symptoms causing the most suspicion of a potential GCA diagnosis consist of jaw claudication, temporal headache (or other type), scalp tenderness and visual disturbance [1]. Genes shared by multiple phenotypes are likely to be particularly relevant to making a diagnosis and could be used as biomarkers for disease activity in the acute setting and potentially predict relapses.

Jaw claudication is often considered the most predictive symptom of GCA; for example, a patient has a nine time greater risk of a positive $\mathrm{TAB}$ when they experience jaw claudication [29]. In CD4 cells of our patient cohort, LAMTOR4, was shared between jaw claudication and temporal headache. This protein is part of the ragulator complex, which is involved in pathways regulating cell size and cell cycle arrest [30]. A gene common to both jaw claudication and visual disturbance is $G Z M B$, otherwise known as Granzyme B enzyme. GZMB is necessary for targeting cell lysis in cell-mediated immune responses and is involved in the activation of cytokine release and cascade of caspases responsible for apoptosis execution. Its involvement has been reported in other autoimmune diseases such as type 1 diabetes and systemic lupus erythematosus [31, 32]. $P P P 1 C B$, linked to vascular smooth muscle contraction pathway [33], was common to patients with jaw claudication and a background history of PMR, which has been shown to increase the risk of GCA [34]. EXTL3, involved in the heparan sulfate biosynthesis pathway and previously associated with syphilis, was expressed in both patients with jaw claudication and fatigue [35].

Multiple genes were associated with temporal and other types of headache in our patients. These included POFUT2 in CD4 cells and SLC35F6, HTD2, ZNF708, KLRC4-KLRK1 and JMJD7 in CD8 cells. These genes have been implicated in cellular defense mechanisms, innate immunity, cell proliferation and apoptosis signaling pathways [36]. One example of great clinical interest is a gene shared by patients with a history of PMR and those experiencing visual disturbances at T1. ETS1, controls lymphocyte differentiation and modulates cytokine and chemokine expression. Low expression levels of ETS1, leading to aberrant lymphocyte differentiation, have been found in systemic lupus erythematosus [37]. ETS1 also has a potential role in the regulation of angiogenesis [38]. ETS1 warrants further functional investigation in relation to its vascular role and as a biomarker for GCA for those patients presenting with PMR.

Three patients died during the study period. The exact cause of death for the three patients is unknown. One patient was being managed by her rural GP as lived rurally. She lived in a nursing home after losing vision in both eyes from GCA. The second patient who died was also bilaterally blind from GCA. She suffered multiple falls and died soon after. The third patient, admitted to hospital for general decline in health, was investigated for possible stroke. She had very poor appetite and died within a few weeks of admission. The bilateral visual loss likely predisposes to poor outcome, whether directly as a sign of disease severity or possibly due to increased likelihood of falls and other morbidity indirectly increasing mortality.

We determined gene correlations with markers of disease prognosis and severity (Table 3 ). Genes in association with poor prognostic outcome markers of GCA, such as blindness, relapses and death could provide useful predictions in the acute setting and could help determine the treatment intensity and length required for those particular patients. We identified genes that overlap between acute phase markers as well as the prognostic markers. For example, temporal headache at T1 as well as bilateral blindness showed significant association with CD8 expression of TCF7, which is important for adaptive $\mathrm{T}$ lymphocyte and innate lymphoid cell regulation [39]. Both these phenotypes were also associated with NUCB2, which encodes Nesfatin-1. NUCB2 is linked to inflammation and coagulopathies, and is correlated with mortality following brain injury [40]. As TCF7 and NUCB2 expression are associated with temporal headache in patients with GCA, these genes could also raise suspicion of poor visual outcome in patients presenting with temporal headache with GCA diagnosis.

We identified 15 genes shared across three phenotypes in CD4 and 16 across CD8 cells (Table 5). In CD4 cells, $S R R T$, a gene associated with cell proliferation [41], was common to four phenotypes: death, fever, and both types of headaches. In CD8, IL32, a member of the cytokine family [42], was common to five phenotypes: a history of PMR, visual disturbance and raised neutrophils at T1, bilateral blindness and death within 12 months. IL32 involvement has been described in vasculitides such as granulomatosis with polyangiitis and anti-neutrophil cytoplasm antibodies (ANCA) associated vasculitis [43, 44]. A previous quantitative gene expression analysis study investigating IL32 in GCA demonstrated a strong and significant up-regulation of IL32 in TAB specimens 
of patients with GCA; in particular it was highly expressed by vascular smooth muscle cells of inflamed arteries and neovessels within inflammatory infiltrates [45]. This study also evaluated circulating CD4+ Th1 lymphocytes by flow cytometry which showed that there was a greater abundance of them in GCA patients than controls and that they produced greater amounts of IL32 [45]. From our study, expression of IL32 in patients presenting with visual disturbance, a history of PMR in the presence of an abnormal neutrophil count, should raise suspicion of GCA diagnosis with poor prognostic outcome. Altered expression of these genes should raise suspicion of GCA diagnosis with poor outcome. Such genes warrant more investigation in the context of GCA as these correlated with not only clinical and biochemical phenotypes but also with prognoses.

The current mainstay treatment of high-dose corticosteroids is effective but is commonly associated with potentially serious complications affecting up to $89 \%$ of those with GCA [3]. Even after successful initial treatment with corticosteroids, GCA relapses in up to two-thirds of patients [46]. As shown by our study, 5 out of 16 patients experienced relapses requiring an increase in steroid dose (Additional file 1: Table S1). Unlike in other autoimmune diseases, most steroid-sparing agents and the use of adjunct agents in GCA [MB1] are not associated with a significant improvement in outcome [46, 47]. Tocilizumab, a humanized monoclonal antibody directed against the IL- 6 receptor, has been found to improve both induction and maintenance of remission in patients with GCA for up to 12 months [48]. However, there is a large side effect profile from Tocilizumab. Interestingly, we did not see DGE for IL6.

\section{Conclusion}

GCA is a devastating disease associated with significant morbidity and mortality. We present the first longitudinal gene expression study undertaken to identify robust transcriptomic biomarkers of GCA.

Our results show cell type-specific transcript expression profiles. We have identified genes potentially implicated in the patho-aetiology of GCA which may uncover important biological pathways for this disease. In addition we have identified novel gene-phenotype associations which, in the acute phase, could act as clinical prognostic markers by providing insight into potential disease severity and therefore guide in initiating appropriate patient management.

Further functional investigation is needed to understand the pathways in which the identified genes play a role in the pathogenesis of GCA and to determine whether the DGE in this study can be translated into the clinical setting as new potential biomarkers to monitor disease activity, predict outcome and assist in finding more effective and safer treatments for GCA.

\section{Additional files}

Additional file 1: Table S1. Cases recruited and attendance for all 6 time points (DOCX $160 \mathrm{~kb}$ )

Additional file 2: Figure S1. Quality control metrics for stored specimens. Representative FACS analysis for FITC bound CD4 (A) and APC bound CD8 cells (B). Panel $\mathbf{C}$ displays the FACS confirmed purity of all specimens, with case and control samples represented by red and blue triangles respectively. (DOCX $100 \mathrm{~kb}$ )

Additional file 3: Figure S2. Effect of batch correction on 195 samples ( 2 samples of the 197 were removed). Three parameters (Flowcell ID, Gender and Ethnicity) were used to remove confounding effects in edgeR. PC1 contributes the greatest amount of variance and is largely attributed to Flowcell ID, which accounts for most of the variance in sequencing experiments. (DOCX $384 \mathrm{~kb}$ )

Additional file 4: Table S2. Ophthalmic clinical summary data (GCA cases, $n=16)$. (DOCX $51 \mathrm{~kb}$ )

Additional file 5: Table S3. General disease outcome and prognostic measures. (DOCX 38 kb)

Additional file 6: Figure S3. Expression levels of the top 500 most variable transcripts in CD4 and CD8 cells, shown for each of 135 samples. Sample groups are indicated by the orange (CD4) and blue (CD8) bars at the top of the heatmap. (DOCX $555 \mathrm{~kb}$ )

Additional file 7: Table S4. Differential expression results CD4. (XLSX 272 kb) Additional file 8: Table S5. Differential expression results CD8. (XLSX 1097 kb) Additional file 9: Table S6. Significant polynomial modelling results. (XLSX 62 kb)

Additional file 10: Table S7. Significant CD4 genes shared by phenotypes at T1. (CSV $4 \mathrm{~kb}$ )

Additional file 11: Table S8. Significant CD8 genes shared by phenotypes at T1. (CSV 5 kb)

\section{Abbreviations}

ATGC: Australian Translational Genomics Centre; CERA: Centre for Eye Research Australia; CPM: Counts-per-million; CRP: C-reactive Protein; DE: Differentially expressed; DGE: Differential gene expression; ESR: Erythrocyte Sedimentation Rate; FACS: Fluorescence-Activated Cell Sorting; FC: fold change; FDR: False Discovery Rate; GCA: Giant Cell Arteritis; IL: Interleukin; MACS: Magnetic-Assisted Cell Sorting; PBMC: Peripheral Blood Mononuclear Cells; PMR: Polymyalgia Rheumatica; RIN: RNA Integrity Number; RVEEH: Royal Victorian Eye and Ear Hospital; T1-6: Time points 1 to 6; WCC: White cell count

\section{Acknowledgements}

We thank all doctors and nurses at the Royal Victorian Eye and Ear Hospital for referring patients to us over the course of this study. We thank the Flow Cytometry Facility at the University of Melbourne Brain Centre for analysing the purity of our cell populations. Mostly, we thank all patients for their time and on-going efforts to participate in this work.

\section{Funding}

This work was supported by funding from Arthritis Australia, the Ophthalmic Research Institute of Australia, and an Australian National Health and Medical Research Council (NHMRC) grant (1083405), an NHMRC Practitioner

Fellowship (AWH, 1103329), NHMRC Career Development Fellowship (JEP, 1107599) an Australian Research Council Future Fellowship (AP,

FT140100047). CERA receives Operational Infrastructure Support from the Victorian Government. The funders had no role in study design, data collection and analysis, decision to publish, or preparation of the manuscript. 


\section{Availability of data and materials}

All data generated during this study are included in this published article and its additional files. The datasets used for analysis during the current study are not publicly available due to patient confidentiality reasons but are available from the corresponding author on reasonable request.

\section{Authors' contributions}

EDS coordinated the study, recruited patients, performed MACS experiments and RNA batch extractions. LC took patients blood samples, performed MACS and liaised with patients to organise follow-up study appointments. KC, AWTY and CC assisted in patient recruitment and MACS. NS referred patients from the neuro-ophthalmology service at the RVEEH and managed patients' medical care. ILS and SH assisted and supervised RNA extractions. LA, SS, BW, LW in MB's laboratory performed the CDNA preparations and subsequent RNA sequencing of samples across the 9 flow cells. SL, AS, KD and JP performed the bioinformatic analysis on the data generated from the RNA sequencing. DM, AP and AH organised funding for this work and designed the overall study. EDS and SL wrote the article. All authors read and approved the final manuscript.

\section{Ethics approval and consent to participate}

Ethics was approved for this study through the Royal Victorian Eye and Ear Hospital (Ethics 11/998H), and all patients provided informed written consent to participate in serial sample collections, and for publication of results.

\section{Consent for publication}

N/A - no individual data is released in this manuscript.

\section{Competing interests}

The authors declare that they have no competing interests.

\section{Publisher's Note}

Springer Nature remains neutral with regard to jurisdictional claims in published maps and institutional affiliations.

\begin{abstract}
Author details
${ }^{1}$ Centre for Eye Research Australia, The University of Melbourne, Royal Victorian Eye \& Ear Hospital, 32 Gisborne Street, East Melbourne 3002 Australia. ${ }^{2}$ Institute for Molecular Bioscience, The University of Queensland, Brisbane 4072, Queensland, Australia. Institute of Health and Biomedical Innovation, Queensland University of Technology, Translational Research Institute, Princess Alexandra Hospital, Brisbane 4102, Queensland, Australia. ${ }^{4}$ Ophthalmology Department at Monash Health, Department of Surgery, School of Clinical Sciences at Monash Health, Melbourne 3168, Victoria, Australia. ${ }^{5}$ Department of Neuro-Ophthalmology, Royal Victorian Eye and Ear Hospital, Melbourne 3002, Victoria, Australia. ${ }^{6}$ Centre for Ophthalmology and Visual Science, The University of Western Australia, Lions Eye Institute, Perth 6009, Western Australia, Australia. ${ }^{7}$ School of Medicine, Menzies Research Institute Tasmania, University of Tasmania, Hobart 7000, Tasmania, Australia.
\end{abstract}

Received: 27 March 2018 Accepted: 26 June 2018

\section{Published online: 23 July 2018}

\section{References}

1. De Smit E, O'Sullivan E, Mackey DA, Hewitt AW. Giant cell arteritis: ophthalmic manifestations of a systemic disease. Graefes Arch Clin Exp Ophthalmol. 2016;254:2291-306.

2. De Smit E, Clarke L, Sanfilippo PG, Merriman TR, Brown MA, Hill CL, et al. Geo-epidemiology of temporal artery biopsy-positive giant cell arteritis in Australia and New Zealand: is there a seasonal influence? RMD Open. 2017; 3:e000531.

3. Dunstan E, Lester SL, Rischmueller M, Dodd T, Black R, Ahern M, et al. Epidemiology of biopsy-proven giant cell arteritis in South Australia. Intern Med J. 2014:44:32-9.

4. Seeliger B, Sznajd J, Robson JC, Judge A, Craven A, Grayson PC, et al. Are the 1990 American College of Rheumatology vasculitis classification criteria still valid? Rheumatology. 2017; https://doi.org/10.1093/rheumatology/ kex075.

5. Carmona FD, Mackie SL, Martín J-E, Taylor JC, Vaglio A, Eyre S, et al. A largescale genetic analysis reveals a strong contribution of the HLA class II region to giant cell arteritis susceptibility. Am J Hum Genet. 2015;96:565-80.
6. Samson M, Ly KH, Tournier B, Janikashvili N, Trad M, Ciudad M, et al. Involvement and prognosis value of CD8(+) T cells in giant cell arteritis. J Autoimmun. 2016;72:73-83.

7. Weyand CM, Liao YJ, Goronzy JJ. The immunopathology of giant cell arteritis: diagnostic and therapeutic implications. J Neuroophthalmol. 2012; 32:259-65.

8. Chaussabel D, Pascual V, Banchereau J. Assessing the human immune system through blood transcriptomics. BMC Biol. 2010;8:84.

9. Lyons PA, Koukoulaki M, Hatton A, Doggett K, Woffendin HB, Chaudhry AN, et al. Microarray analysis of human leucocyte subsets: the advantages of positive selection and rapid purification. BMC Genomics. 2007;8:64.

10. Bray NL, Pimentel H, Melsted P, Pachter L. Near-optimal probabilistic RNAseq quantification. Nat Biotechnol. 2016;34:525-7.

11. Robinson MD, McCarthy DJ, Smyth GK. edgeR: a Bioconductor package for differential expression analysis of digital gene expression data. Bioinformatics. 2010;26:139-40.

12. Kamburov A, Stelzl U, Lehrach H, Herwig R. The ConsensusPathDB interaction database: 2013 update. Nucleic Acids Res. 2013;41:D793-800.

13. Benjamini Y, Hochberg Y. On the adaptive control of the false discovery rate in multiple testing with independent statistics. J Educ Behav Stat. 2000;25:60.

14. Buechler C, Ritter M, Orsó E, Langmann T, Klucken J, Schmitz G. Regulation of scavenger receptor CD163 expression in human monocytes and macrophages by pro- and antiinflammatory stimuli. J Leukoc Biol. 2000;67:97-103.

15. Tippett E, Cheng W-J, Westhorpe C, Cameron PU, Brew BJ, Lewin SR, et al. Differential expression of CD163 on monocyte subsets in healthy and HIV-1 infected individuals. PLoS One. 2011;6:e19968.

16. El-Dairi MA, Chang L, Proia AD, Cummings TJ, Stinnett SS, Bhatti MT. Diagnostic algorithm for patients with suspected Giant cell arteritis. J Neuroophthalmol. 2015:35:246-53.

17. Abramson N, Melton B. Leukocytosis: basics of clinical assessment. Am Fam Physician. 2000;62:2053-60

18. Cao M, Xu W, Yu J, Zheng H, Tan X, Li L, et al. Up-regulation of SGTB is associated with neuronal apoptosis after neuroinflammation induced by lipopolysaccharide. J Mol Histol. 2013;44:507-18.

19. Lassaunière $R$, Shalekoff $S$, Tiemessen CT. A novel FCGR3A intragenic haplotype is associated with increased FcyRIIla/CD16a cell surface density and population differences. Hum Immunol. 2013:74:627-34.

20. Chen S, Wen X, Li J, Li Y, Li L, Tian X, et al. Association of FCGR2A/FCGR3A variant rs2099684 with Takayasu arteritis in the Han Chinese population. Oncotarget. 2017; https://doi.org/10.18632/oncotarget.12738.

21. Loupy A, Duong Van Huyen JP, Hidalgo L, Reeve J, Racapé M, Aubert O, et al. Gene expression profiling for the identification and classification of antibody-mediated heart rejection. Circulation. 2017;135:917-35.

22. Lefaucheur C, Viglietti D, Hidalgo LG, Ratner LE, Bagnasco SM, Batal I, et al. Complement-activating anti-HLA antibodies in kidney transplantation: allograft gene expression profiling and response to treatment. J Am Soc Nephrol. 2017; https://doi.org/10.1681/ASN.2017050589.

23. Moestrup SK, Møller HJ. CD163: a regulated hemoglobin scavenger receptor with a role in the anti-inflammatory response. Ann Med. 2004; 36:347-54.

24. Samson M, Corbera-Bellalta M, Audia S, Planas-Rigol E, Martin L, Cid MC, et al. Recent advances in our understanding of giant cell arteritis pathogenesis. Autoimmun Rev. 2017;16:833-44.

25. Ding $D$, Song $Y$, Yao $Y$, Zhang $S$. Preoperative serum macrophage activated biomarkers soluble mannose receptor (sMR) and soluble haemoglobin scavenger receptor (sCD163), as novel markers for the diagnosis and prognosis of gastric cancer. Oncol Lett. 2017;14:2982-90.

26. Rødgaard-Hansen S, St George A, Kazankov K, Bauman A, George J, Grønbæk H, et al. Effects of lifestyle intervention on soluble CD163, a macrophage activation marker, in patients with non-alcoholic fatty liver disease. Scand J Clin Lab Invest. 2017;77:498-504.

27. Pranzatelli MR, Tate ED, McGee NR. Microglial/macrophage markers CHI3L1, sCD14, and SCD163 in CSF and serum of pediatric inflammatory and noninflammatory neurological disorders: a case-control study and reference ranges. J Neurol Sci. 2017;381:285-90.

28. Stilund M, Reuschlein A-K, Christensen T, Møller HJ, Rasmussen PV, Petersen T. Soluble CD163 as a marker of macrophage activity in newly diagnosed patients with multiple sclerosis. PLoS One. 2014;9:e98588.

29. Hayreh SS. Masticatory muscle pain: an important indicator of giant cell arteritis. Spec Care Dentist. 1998;18:60-5. 
30. Stoesser GEMBL. Nucleotide sequence database (EMBL-Bank, EMBL database). Dictionary of Bioinformatics and Computational Biology. 2004;

31. Cullen SP, Brunet M, Martin SJ. Granzymes in cancer and immunity. Cell Death Differ. 2010;17:616-23.

32. Joeckel LT, Bird PI. Blessing or curse? Proteomics in granzyme research. Proteomics Clin Appl. 2014;8:351-81.

33. Takaki T, Montagner M, Serres MP, Le Berre M, Russell M, Collinson L, et al. Actomyosin drives cancer cell nuclear dysmorphia and threatens genome stability. Nat Commun. 2017;8:16013.

34. Buttgereit F, Dejaco C, Matteson EL, Dasgupta B. Polymyalgia Rheumatica and Giant cell arteritis: a systematic review. JAMA. 2016;315:2442-58.

35. Volpi S, Yamazaki Y, Brauer PM, van Rooijen E, Hayashida A, Slavotinek A, et al. EXTL3 mutations cause skeletal dysplasia, immune deficiency, and developmental delay. J Exp Med. 2017;214:623-37.

36. GeneCards. Encyclopedia of Genetics, Genomics, Proteomics and Informatics; 2008. p. 761-1.

37. Garrett-Sinha LA, Kearly A, Satterthwaite AB. The role of the transcription factor Ets1 in lupus and other autoimmune diseases. Crit Rev Immunol. 2016;36:485-510.

38. Yordy JS, Moussa O, Pei H, Chaussabel D, Li R, Watson DK. SP100 inhibits ETS1 activity in primary endothelial cells. Oncogene. 2005;24:916-31.

39. De Obaldia ME, Bhandoola A. Transcriptional regulation of innate and adaptive lymphocyte lineages. Annu Rev Immunol. 2015;33:607-42.

40. Wu G-Q, Chou X-M, Ji W-J, Yang X-G, Lan L-X, Sheng Y-J, et al. The prognostic value of plasma nesfatin-1 concentrations in patients with traumatic brain injury. Clin Chim Acta. 2016:458:124-8.

41. Ashrafi M, Sebastian A, Shih B, Greaves N, Alonso-Rasgado T, Baguneid M, et al. Whole genome microarray data of chronic wound debridement prior to application of dermal skin substitutes. Wound Repair Regen. 2016;24:870-5.

42. Kim S-H, Han S-Y, Azam T, Yoon D-Y, Dinarello CA. Interleukin-32: a cytokine and inducer of TNFalpha. Immunity. 2005;22:131-42.

43. Bae S, Kim Y-G, Choi J, Hong J, Lee S, Kang T, et al. Elevated interleukin-32 expression in granulomatosis with polyangiitis. Rheumatology. 2012;51: 1979-88.

44. Csernok E, Holle JU, Gross WL. Proteinase 3, protease-activated receptor-2 and interleukin-32: linking innate and autoimmunity in Wegener's granulomatosis. Clin Exp Rheumatol. 2008;26:S112-7.

45. Ciccia F, Alessandro R, Rizzo A, Principe S, Raiata F, Cavazza A, et al. Expression of interleukin-32 in the inflamed arteries of patients with giant cell arteritis. Arthritis Rheum. 2011;63:2097-104.

46. Labarca C, Koster MJ, Crowson CS, Makol A, Ytterberg SR, Matteson EL, et al. Predictors of relapse and treatment outcomes in biopsy-proven giant cell arteritis: a retrospective cohort study. Rheumatology. 2016;55:347-56.

47. Yates M, Loke YK, Watts RA, MacGregor AJ. Prednisolone combined with adjunctive immunosuppression is not superior to prednisolone alone in terms of efficacy and safety in giant cell arteritis: meta-analysis. Clin Rheumatol. 2014;33:227-36.

48. Roberts J, Clifford A. Update on the management of giant cell arteritis. Ther Adv Chronic Dis. 2017:8:69-79.

\section{Ready to submit your research? Choose BMC and benefit from:}

- fast, convenient online submission

- thorough peer review by experienced researchers in your field

- rapid publication on acceptance

- support for research data, including large and complex data types

- gold Open Access which fosters wider collaboration and increased citations

- maximum visibility for your research: over $100 \mathrm{M}$ website views per year

At BMC, research is always in progress.

Learn more biomedcentral.com/submissions 